\title{
Noise Properties of Dual Mach-Zehnder Interferometers employing Narrowband Fiber ASE Sources
}

\author{
Hyo Sang Kim, Ronald P. H. Haaksman, Trevor P. Newson, and David J. Richardson \\ Optoelectronics Research Centre, University of Southampton, Southampton SO17 1BJ, UK \\ Tel:+44-1703-593141, Fax:+44-1703-593142, Email: hsk@orc.soton.ac.uk
}

\begin{abstract}
We report a detailed study of the noise properties of dual Mach-Zehnder interferometers interrogated with narrowband $(<15 \mathrm{GHz})$ fiber ASE sources. A minimum detectable phase of $62 \mu \mathrm{rad} / \sqrt{\mathrm{Hz}}$ is obtained for practical values $(\sim 1 \mathrm{~cm})$ of length mismatch using a simple heterodyne technique and optimized configuration.
\end{abstract}

\section{Introduction}

The development of WDM, and in particular fiber Bragg grating technology, driven by the telecommunications industry, will have a major impact in the area of optical fiber sensors. One can now realistically envisage large arrays of sensing interferometers in which each individual sensor, or subset of sensors, is readily demultiplexed in the wavelength domain. Such arrays can be used for example for acoustic, dynamic strain, electric field, and magnetic field sensing. The development of practical interferometric sensor interrogation techniques which allow dense wavelength multiplexing whilst at the same time offering high sensitivity and low cross-talk performance is thus an area of significant commercial interest.

The most obvious approach is to use a conventional narrowband laser source (e.g. semiconductor DFB laser) and the coherence multiplexing method ${ }^{1,2}$. However, this technique is limited by phase-fluctuation induced intensity noise ${ }^{3}$. The optical gating method has been used to avoid this source of noise, and indeed high levels of sensitivity have been demonstrated ${ }^{4}$. However, the need to adopt a gating approach adds considerable complexity to the system and can lead to severe cross-talk problems if the optical gating is anything other than perfect.

The use of a broadband (superfluorescent) source removes the need for optical gating and has also been shown to achieve high sensitivity. However, this approach is not practical for fiber sensor arrays since the path length matching between the reference and sensing interferometers needs to be to within the coherence length of the source $(<1 \mathrm{~mm})$.

In this paper, we investigate the use of narrowband ASE sources for the interrogation of fiber interferometers in the limit that the coherence length of the source is greater than $1 \mathrm{~cm}$, which we consider to be the minimum practical length mismatch. The attractive features of this approach are that the bandwidths required $(<15 \mathrm{GHz})$ allow for a high density of multiplexing and are commensurate with the use of established 100 and $200 \mathrm{GHz}$ (ITU standard) WDM technology. Moreover, since optical gating is not required the approach should allow for simple demodulation schemes with good cross talk performance. The fundamental issue concerning this approach is that of excess photon noise which scales in inverse proportion to bandwidth for a thermal source and becomes the dominant form of source noise in the regime we are investigating. To assess the limits imposed by this form of noise we investigate the noise properties and performance of dual fiber Mach-Zehnder interferometers interrogated with narrowband fiber ASE sources. Our experiments show that these sources do behave exactly as thermal sources, and that excess photon noise does indeed limit the overall system performance. However, we demonstrate that the interferometer has a pronounced impact on the final system noise distribution, and that with an optimized system design and heterodyne technique, a good phase resolution of $\sim 60 \mu \mathrm{rad} / / \mathrm{Hz}$ can be achieved for practical length mismatches.

\section{ASE source noise}

Initially we investigated the noise properties of the narrowband ASE source we developed for our experiments. The experimental configuration is shown in Fig. 1. The first Erbium-doped fiber amplifier(EDFA-1) was used as a broadband optical source. A (tunable) filter with a $3 \mathrm{~dB}$ bandwidth of $1.3 \mathrm{~nm}$ and a second EDFA (EDFA-2) were used to increase the spectral power density around the center-wavelength of a fiber Bragg grating (FBG), whose reflectivity profile was used to define the final source linewidth and spectral shape. The narrowband ASE 


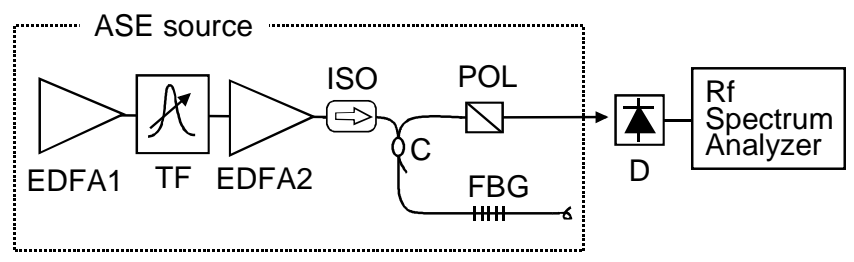

Fig. 1: Experimental setup for the measurement of optical source noise spectrum.

EDFA's: Erbium-doped fiber amplifiers, TF: tunable filter, POL: polarizer, ISO: isolator, C: 50:50 coupler, FBG: fiber Bragg grating, D: detector.
Table 1: Characteristics of fiber Bragg gratings

\begin{tabular}{|c|c|r|r|}
\hline \multirow{2}{*}{ ID } & \multirow{2}{*}{$\begin{array}{c}\text { Center } \\
\text { wavelength } \\
(\mathrm{nm})\end{array}$} & \multicolumn{2}{|c|}{ FWHM } \\
\cline { 3 - 4 } & $(\mathrm{pm})$ & $(\mathrm{GHz})$ \\
\hline FBG1 & 1559.6 & 18 & 2.2 \\
\hline FBG2 & 1545.8 & 45 & 5.6 \\
\hline FBG3 & 1529.5 & 60 & 7.7 \\
\hline FBG4 & 1529.3 & 81 & 10.4 \\
\hline FBG5 & 1529.3 & 115 & 14.7 \\
\hline
\end{tabular}

was polarized after passing through the $3 \mathrm{~dB}$ output-coupler. In order to vary the linewidth of the output we simply changed the FBG. Five different narrowband gratings were available with the characteristics shown in table 1. Noise spectra were measured as a function of power and linewidth using an InGaAs optical receiver with a scale factor of $18 \mathrm{~V} / \mathrm{mW}$ and an RF spectrum analyzer.

We first measured the spectral noise power at three different frequencies $(1,10,100 \mathrm{MHz})$ as a function of optical power for fixed source linewidth (defined by grating FBG3). The experimental results are shown in Fig.2, where we see that the spectral noise power was flat across the measured frequency range due to the relatively broad linewidth of the source $(7.7 \mathrm{GHz})$ compared to the detection bandwidth $(200 \mathrm{MHz})$. From these measurements the relative intensity noise of the source was calculated to be $-97.7 \mathrm{~dB} / \mathrm{Hz}$. Superposed on Fig 2 is the detector noise, and the theoretically calculated ASE source noise (excess photon noise), shot noise, and total noise. The source noise calculations were based on a Gaussian random process model ${ }^{5,6}$, and incorporated the experimentally measured lineshape as defined by FBG3. The experiments are seen to be in excellent agreement with theory and confirm that excess photon noise is the dominant form of noise at any appreciable power level for these sources. In Fig. 3 we plot the measured and calculated spectral noise power at $10 \mathrm{MHz}$ as a function of linewidth for optical powers of $-20 \mathrm{dBm}$ and $-23 \mathrm{dBm}$. The results clearly show that the spectral noise power is inversely proportional to the linewidth as expected for a thermal source.

\section{Noise properties of heterodyne, Dual Mach-Zehnder Interferometer.}

It is well established theoretically that the filtering effect of an interferometer serves to redistribute source noise in the frequency domain ${ }^{7}$. The exact manner in which the noise is redistributed is a function of the precise configuration and implementation of the system. It is also clear that any practical, high-sensitivity, interferometer system is going to require some form of signal modulation to avoid the high environmental noise within the low frequency range. One general method is to use a heterodyne technique. For these reasons we chose to examine the noise distribution properties of dual fiber Mach-Zehnder interferometer with a frequency shifter in one of the

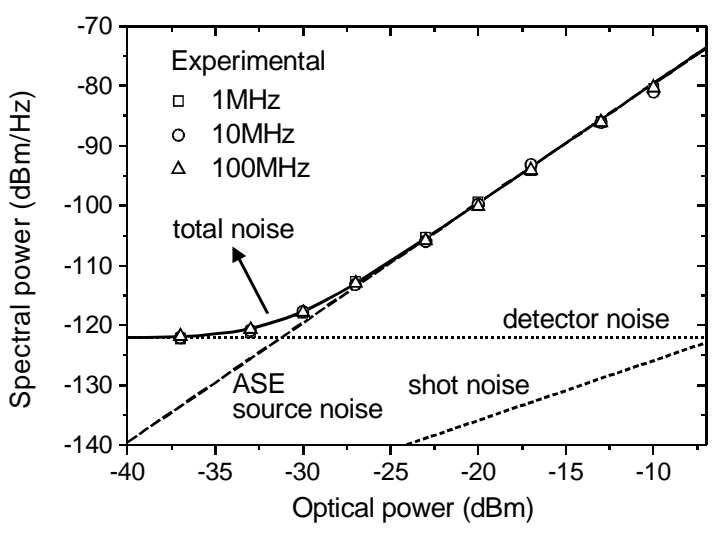

Fig. 2: Spectral noise power vs. optical power. The three symbols are the measured noise at the frequency of 1 $\mathrm{MHz}(\square), 10 \mathrm{MHz}(\mathrm{O})$, and $100 \mathrm{MHz}(\triangle)$ ). The lines (except the detector noise) are obtained from theory.

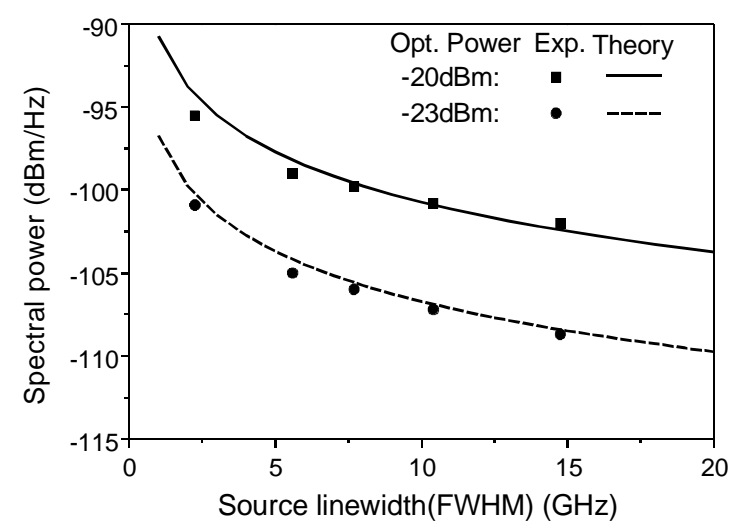

Fig. 3: Spectral noise power vs. source linewidth. The measurements were carried out at $10 \mathrm{MHz}$ 


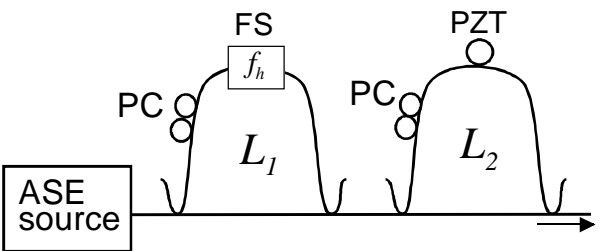

(a)

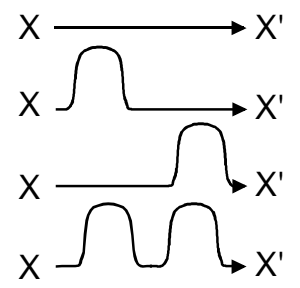

(b)

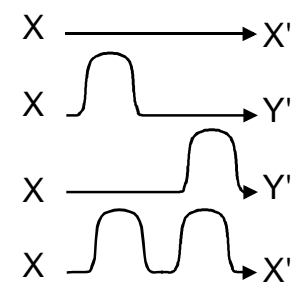

(c)

Fig. 4: Dual Mach-Zehnder interferometer using a frequency shifter

(a) Schematics (b) Polarization Preserving case(PP) (c) Polarization Flipping case(PF).

FS: frequency shifter, PC: Polarization controller, $L_{\mathrm{i}}$ 's : Length imbalance.

arms of the first (reference) Mach-Zehnder to allow for heterodyne detection (see Fig.4a). Whilst the detailed results presented below are specific to this particular choice of system we wish to point out that the general features and conclusions of this work are appropriate to other interferometric arrangements.

Since the system noise distribution depends on the birefringence within the interferometers we incorporated polarization controllers within each interferometer, and made noise measurements for two extreme cases. In the first instance we ensured that the light arriving from each arm at each of the interferometer output couplers was co-polarized (polarization preserving case), which is illustrated in Fig. 4 (b). In the second case we ensured that the polarization states of the light passing through the two paths in each interferometer were made orthogonal to each other at the output couplers (polarization flipping (PF-) case, Fig 4 (c)). As will be seen later the PF configuration gives the optimum noise performance.

The delay lengths of the two single Mach Zehnder interferometers, $L_{1}$ and $L_{2}$, were $\sim 26.52 \mathrm{~m}$, a value chosen to minimize the noise around the heterodyne frequency of $27.12 \mathrm{MHz}$ as defined by the frequency shifter. A PZT phase modulator was inserted in one of the arms of the second interferometer to allow us to apply a well-defined level of phase modulation. We performed experiments for length mismatches of $1.3 \mathrm{~mm}$ and $10.9 \mathrm{~mm}$. The length mismatches were estimated from the periods of the measured noise spectra of the individual interferometers to a resolution of $\sim 1.0 \mathrm{~mm}$. The averaged optical output power was set to be $-23 \mathrm{dBm}$ throughout.

Fig 5 shows the noise spectra for the source linewidth of $7.7 \mathrm{GHz}$ obtained from both theory and experiment for the different polarization settings and for a length mismatch of $1.3 \mathrm{~mm}$. The coherence length of the source, defined as the length mismatch where the visibility falls to half that of the matched interferometer, is $12.4 \mathrm{~mm}$. The noise redistribution is seen to be very pronounced. Remember, that as shown in Fig.2, the noise level of the source itself at the $-23 \mathrm{dBm}$ power level is $-105.6 \mathrm{dBm} / \mathrm{Hz}$ and is independent of frequency in the $0-100 \mathrm{MHz}$ frequency range. The periodic structure in the noise spectrum is due to the filtering effect of the interferometers. The period $(7.75 \mathrm{MHz})$ is the inverse of the elapsed time for light to propagate though the 26.5 meter-long fiber.

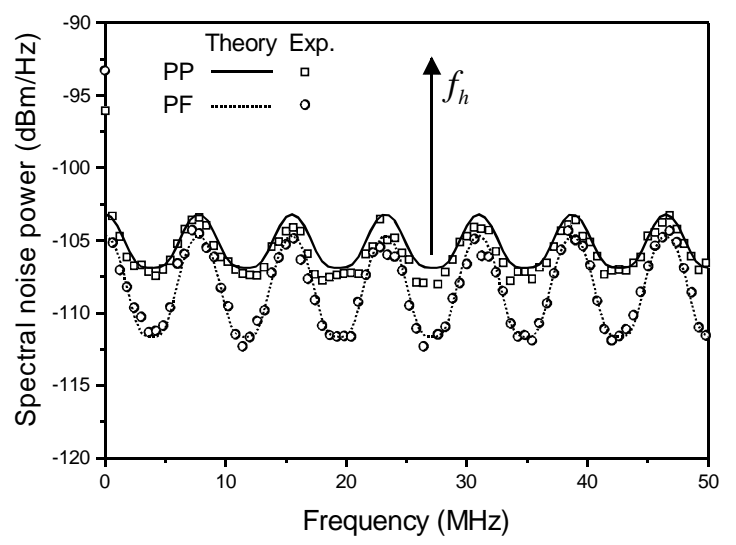

Fig. 5: Noise spectra for dual MZ using heterodyne method.

PP: Polarization Preserving case, PF: Polarization Flipping case, $f_{\mathrm{h}}$ : heterodyne signal.

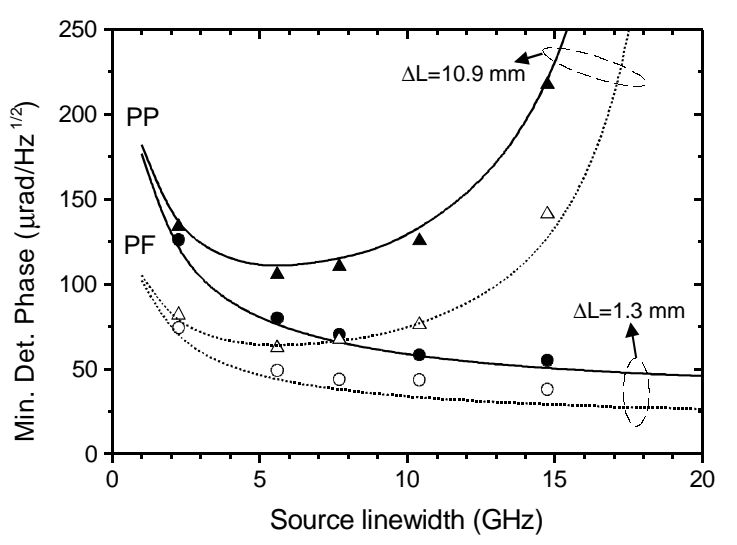

Fig. 6: Minimum detectable phase vs. source linewidth for different length mismatches $(1.3 \mathrm{~mm}$ and $10.9 \mathrm{~mm})$. PP: Polarization Preserving case, PF: Polarization Flipping case. The symbols are experimental results, lines are from theory. 
The noise level of the polarization flipping case $(\mathrm{PF})$ is seen to be smaller than that of the polarization preserving case(PP) over the full detection bandwidth, falling to $\sim-112 \mathrm{dBm} / \mathrm{Hz}$ at the spectral minima. In both cases the signal power was the same for a fixed level of phase modulation. The signal to noise ratio is therefore substantially higher $(\sim 5 \mathrm{~dB})$ for the PF case. Similar patterns and levels of noise redistribution were obtained for other values of source linewidth and length mismatch.

The minimum detectable phase was measured by applying a known level of phase modulation to the PZT and observing the heterodyne signal and noise floor on an RF spectrum analyzer. Measurements were made as a function of source linewidth for the two length mismatches of $1.3 \mathrm{~mm}$ and $10.9 \mathrm{~mm}$, and for the different polarization settings(PP \& PF). The results are shown in Fig.6, superposed are theoretical curves from our detailed noise analysis of the system. Good agreement between experiment and theory is obtained. From Fig. 6 it is seen that a minimum detectable phase of $62 \mu \mathrm{rad} / \sqrt{ } \mathrm{Hz}$ is achieved for the practical length mismatch of $10.9 \mathrm{~mm}$. This minimum value is obtained for a linewidth of $\sim 5 \mathrm{GHz}$. At narrower source linewidths the increased source noise reduces the sensitivity, whilst at broader linewidths the rapidly reducing signal visibility limits performance. For smaller, (but impractical), length mismatches significantly lower minimum detectable phases can be obtained, values as low as $25 \mu \mathrm{rad} / \sqrt{ } \mathrm{Hz}$ have been achieved in our experiments. These results provide important information as to how to design an optimized system for any level of practical limitation on length mismatch, source linewidth, sensor length etc.

\section{Conclusion}

We have investigated both experimentally and theoretically the noise characteristics and sensitivity limits of dual fiber Mach-Zehnder interferometers interrogated using narrowband fiber ASE sources and a heterodyne technique. We have shown that the system performance is limited by the excess photon noise of the source and that the detailed system noise spectrum is dependent on the filtering effect and birefringence in the optical paths of the interferometer. By utilizing these noise redistribution effects and optimizing the source linewidth, we have shown that it is possible to reduce the noise level in the signal frequency region and have achieved a minimum detectable phase of $62 \mu \mathrm{rad} / \sqrt{ } \mathrm{Hz}$, for a path length mismatch of $\sim 1 \mathrm{~cm}$. We consider the approach we have demonstrated to be highly practical, providing good phase resolution whilst remaining consistent with interferometer manufacturing tolerances and requiring relatively simple demodulation methods.

\section{Acknowledgment}

The authors wish to thank Dr. Malcolm Varnham of Sensor Dynamics Ltd. (UK) for useful discussions and Mr. Morten Ibsen for the supply of gratings used in these experiments. This work is funded in part by the UK EPSRC grant No. GR/L87057, Sensor Dynamics Ltd. (UK) and Geosensor Corp. (USA).

\section{References}

[1] J. L. Brooks, R. H. Wentworth, R. C. Youngquist, M. Tur, B. Y. Kim, and H. J. Shaw, "Coherence multiplexing of fiber-optic interferometric sensors," J. Lightwave Technol., vol. LT-3, pp.1062-1071, 1985.

[2] K. Iiyama and K. Hayashi, "Frequency domain detection of coherence multiplexed sensor signals by using an optical loop with a frequency shifter,” J. Lightwave Technol., vol. 15, pp.2069-2075, 1997.

[3] B. Moslehi, "Noise power spectra of optical two-beam interferometers induced by the laser phase noise," $J$. Lightwave Technol., vol. LT-4, pp.1704-1709, 1986.

[4] J. L. Brooks, M. Tur, B. Y. Kim, K. A. Felser, and H. J. Shaw, "Fiber-optics interferometric sensor arrays with freedom from source phase-induced noise," Opt. Lett., vol. 11, pp.473-375, 1986.

[5] J. W. Goodman, Statistical Optics, New York: John Wiley \& Sons, 1985.

[6] M. Tur, E. Shafir, and K. Bløtekjaer, "Source-induced noise in optical systems driven by low-coherence sources," J. Lightwave Technol., vol. 8, pp.183-189, 1990.

[7] K. P. Jackson, S. A. Newton, B. Moslehi, M. Tur, C. C. Culter, J. W. Goodman, and H. J. Shaw, "Optical fiber delay-line signal processing," IEEE Trans. Microwave Theory Tech., vol. MTT-33, pp.193-208, 1985. 